\title{
A SINC-HUNTER QUADRATURE RULE FOR CAUCHY PRINCIPAL VALUE INTEGRALS
}

\author{
BERNARD BIALECKI
}

\begin{abstract}
A Sinc function approach is used to derive a new Hunter type quadrature rule for the evaluation of Cauchy principal value integrals of certain analytic functions. Integration over a general arc in the complex plane is considered. Special treatment is given to integrals over the interval $(-1,1)$. It is shown that the quadrature error is of order $O\left(e^{-c \sqrt{N}}\right)$, where $N$ is the number of nodes used, and where $c$ is a positive constant which is independent of $N$. An application of the rule to the approximate solution of Cauchy singular integral equations is also discussed. Numerical examples are included to illustrate the performance of the rule.
\end{abstract}

\section{INTRODUCTION AND SUMMARY}

Let $\Gamma$ be an arc in the complex plane, and let $a$ and $b$ denote the endpoints of $\Gamma$. It is assumed that neither $a$ nor $b$ belongs to $\Gamma$. Let $D$ be a domain containing $\Gamma$ such that $a$ and $b$ are boundary points of $D$. Suppose that a function $F$, defined and integrable on $\Gamma$, has an analytic extension into $D(F$ may become unbounded at either $a$ or $b$ ). For $\lambda$ in $\Gamma$, the Cauchy principal value integral is defined by

$$
\mathscr{H}(F ; \lambda) \equiv f_{\Gamma} \frac{F(z)}{z-\lambda} d z=\lim _{\varepsilon \rightarrow 0^{+}} \int_{\Gamma_{\varepsilon}} \frac{F(z)}{z-\lambda} d z,
$$

where $\Gamma_{\varepsilon}$ is the part of $\Gamma$ obtained by deleting from $\Gamma$ all those points which are within a distance $\varepsilon$ from $\lambda$.

In this paper a Sinc function approach is used to derive a new Hunter type quadrature rule for the evaluation of (1.1). Several Sinc function methods of computing (1.1) have been previously proposed in $[12,13,6]$. The quadrature rule of this note is more accurate than those of $[12,13,6]$, and, moreover, it is well suited for direct methods of solving Cauchy singular integral equations (see $[6,1,3]$ for indirect methods based on a regularization process).

If the function $F$ appearing in (1.1) has singularities at $a$ or $b$, then it is sometimes possible to rewrite $F$ as $w G$, where the bad behavior of $F$ is incorporated into the weight function $w$, and where $G$ is a smooth function.

Received March 24, 1989.

1980 Mathematics Subject Classification (1985 Revision). Primary 65D30, 45 E05.

Key words and phrases. Gauss quadratures, Cauchy singular integral equations. 
In such cases, Gauss type quadrature rules (see, e.g., $[10,4]$ ) or rules based on piecewise-polynomial approximation of $G$ (see, e.g., [8]) can be used to evaluate (1.1). However, these methods require a considerable amount of computational work for nonclassical weight functions $w$. Moreover, if higher-order derivatives of $G$ are unbounded at $a$ or $b$, then Gauss quadratures will, in general, exhibit a slow rate of convergence. In comparison, the quadrature rule presented here does not make use of the splitting $F=w G$. The rule is very easy to implement and its error converges to zero at the rate $O\left(e^{-c N^{1 / 2}}\right)$, where $N$ is the number of nodes used, and where $c$ is a positive constant which is independent of $N$.

The Sinc-Hunter quadrature rule for a general arc $\Gamma$ is developed in $\S 2$. The derivation of the rule and the corresponding error bound is based on the results in [2]. In $\S 3$, the important special case of $\Gamma=(-1,1)$ is discussed in more detail. An application of the rule to the approximate solution of Cauchy singular integral equations is considered in $\S 4$. It is demonstrated that in some cases, quadrature methods based on the present rule yield better accuracy than that achieved by solving Cauchy singular integral equations via Gauss type quadrature methods. Finally, the results of numerical tests are presented in $\S 5$.

\section{QuADRATURE RULE OVER A GENERAL ARC}

In this section a new Sinc quadrature rule is derived for the approximate evaluation of Cauchy principal value integrals.

Throughout the paper, the letters $\mathbb{Z}, \mathbb{R}$, and $\mathbb{C}$ denote respectively the set of all integers, the set of real numbers, and the set of complex numbers, i.e., $\mathbb{Z}=\{n: n=0, \pm 1, \ldots\}, \mathbb{R}=(-\infty, \infty)$, and $\mathbb{C}=\{z=x+i y: x \in \mathbb{R}$, $y \in \mathbb{R}\}$. For $d>0$, the domain $D_{d}$ is defined by

$$
D_{d}=\{z \in \mathbb{C}:|\operatorname{Im} z|<d\} .
$$

In this section the following conditions are assumed to be satisfied.

Assumption 2.1. Let $D$ be a simply connected domain in the complex plane $\mathbb{C}$, and let $a$ and $b(a \neq b)$ be points on the boundary $\partial D$ of $D$ (see Figure 1).

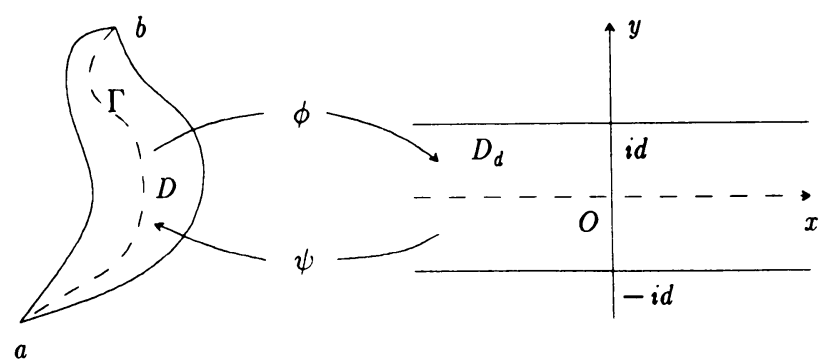

Figure 1. The domains $D$ and $D_{d}$ 
Let $D_{d}$ be as in (2.1), and let $\phi$ be a conformal map of $D$ onto $D_{d}$, such that $\phi(a)=-\infty$, and $\phi(b)=\infty$. Let $\psi$ denote the inverse map of $\phi$, and let

$$
\Gamma=\{\psi(x): x \in \mathbb{R}\},
$$

where the direction along $\Gamma$ is that from $a$ to $b$.

The following definition is fundamental for this paper.

Definition 2.1. Let $B(D)$ denote the family of all functions $F$ that are analytic in $D$, such that

$$
\int_{\psi\left(L_{x}\right)}|F(z) d z| \rightarrow 0 \quad \text { as } x \rightarrow \pm \infty
$$

and such that

$$
N(F, D) \equiv \liminf _{y \rightarrow d^{-}} \int_{\psi\left(L_{y}\right)}|F(z) d z|<\infty,
$$

where for $x \in \mathbb{R}$ and $0<y<d$,

$$
\begin{gathered}
L_{x}=\{z=x+i v:-d<v<d\}, \\
L_{y}=\{z=u \pm i y: u \in \mathbb{R}\} .
\end{gathered}
$$

The main result of this section is given in the following theorem.

Theorem 2.1. Assume $F \in B(D)$ and consider $\mathscr{H}(F ; \lambda)$ defined by (1.1). For $h>0$, let $z_{n}=\psi(n h), n \in \mathbb{Z}$, and let

$$
\mathscr{H}_{-\infty}^{\infty}(F ; \lambda)=\left\{\begin{array}{c}
h \sum_{n \in \mathbb{Z}} \frac{F\left(z_{n}\right)}{\phi^{\prime}\left(z_{n}\right)\left(z_{n}-\lambda\right)}+\pi \cot \left[\frac{\pi}{h} \phi(\lambda)\right] F(\lambda) \\
\text { if } \lambda \neq z_{l} \text { for all } l \in \mathbb{Z}, \\
h \sum_{\substack{n \in \mathbb{Z} \\
n \neq l}} \frac{F\left(z_{n}\right)}{\phi^{\prime}\left(z_{n}\right)\left(z_{n}-\lambda\right)}-\frac{h \phi^{\prime \prime}(\lambda)}{2\left[\phi^{\prime}(\lambda)\right]^{2}} F(\lambda)+\frac{h}{\phi^{\prime}(\lambda)} F^{\prime}(\lambda) \\
\text { if } \lambda=z_{l} \text { for some } l \in \mathbb{Z} .
\end{array}\right.
$$

Then

$$
\left|\mathscr{H}(F ; \lambda)-\mathscr{H}_{-\infty}^{\infty}(F ; \lambda)\right| \leq N(F, D, \lambda) \frac{e^{-2 \pi d / h}}{1-e^{-2 \pi d / h}}
$$

where

$$
N(F, D, \lambda) \equiv \liminf _{y \rightarrow d^{-}} \int_{\psi\left(L_{y}\right)}\left|\frac{F(z)}{z-\lambda} d z\right|,
$$

and where $L_{y}$ in (2.9) is defined by (2.6).

Proof. First assume that $\lambda \neq z_{l}$ for all $l \in \mathbb{Z}$. Choose $a_{1}, a_{2}$ in $D \backslash \Gamma$ so that $a_{1}$ lies on the left-hand side of $\Gamma$ and $a_{2}$ is to the right of $\Gamma$. Let 
$F_{1}$ and $F_{2}$ be two functions defined by $F_{1}(z)=F(z) /\left(z-a_{1}\right)$ and $F_{2}(z)=$ $F(z) /\left(z-a_{2}\right)$, respectively. Note that $F_{1}$ and $F_{2}$ have first-order poles at $a_{1}$ and $a_{2}$, respectively, and that $\operatorname{Res}\left(F_{1} ; a_{1}\right)=F\left(a_{1}\right)$ and $\operatorname{Res}\left(F_{2} ; a_{2}\right)=F\left(a_{2}\right)$, where $\operatorname{Res}(F ; a)$ denotes the residue of $F$ at $a$. Moreover, it is easy to see that $F_{1}, F_{2}$ satisfy all hypotheses of Theorem 3.1 of [2]. Hence, (3.15) and the estimate (3.18) of [2] give

$$
\begin{aligned}
& \mid \int_{\Gamma} \frac{F(z)}{z-a_{1}} d z+\int_{\Gamma} \frac{F(z)}{z-a_{2}} d z-h \sum_{n \in \mathbb{Z}} \frac{F\left(z_{n}\right)}{\phi^{\prime}\left(z_{n}\right)}\left\{\frac{1}{z_{n}-a_{1}}+\frac{1}{z_{n}-a_{2}}\right\} \\
& -\pi\left\{F\left(a_{1}\right) \frac{e^{i \pi \phi\left(a_{1}\right) / h}}{\sin \left[\pi \phi\left(a_{1}\right) / h\right]}+F\left(a_{2}\right) \frac{e^{-i \pi \phi\left(a_{2}\right) / h}}{\sin \left[\pi \phi\left(a_{2}\right) / h\right]}\right\} \mid \\
& \leq\left\{N\left(F_{1}, D\right)+N\left(F_{2}, D\right)\right\} \frac{e^{-2 \pi d / h}}{1-e^{-2 \pi d / h}} .
\end{aligned}
$$

Letting $a_{1}, a_{2} \rightarrow \lambda,(2.8)$ follows from (2.10) and the Plemelj formula (see, e.g., (17.4) in [11]).

Now assume that $\lambda=z_{l}$ for some $l \in \mathbb{Z}$. This case can be reduced to the one considered above. Let $\lambda^{\prime} \in \Gamma$ be near $\lambda$, and $\lambda^{\prime} \neq \lambda$. Then (2.8) holds true for $\lambda^{\prime}$ in place of $\lambda$ and $\mathscr{H}_{-\infty}^{\infty}\left(F ; \lambda^{\prime}\right)$ given by the first expression in (2.7). The second formula in (2.7), and (2.8) for $\lambda=z_{l}$ are now obtained by taking the limit as $\lambda^{\prime} \rightarrow \lambda$.

Using a new class of Sinc functions, Elliott and Stenger have derived a different quadrature rule for the approximate evaluation of $\mathscr{H}(F ; \lambda)$. However, their rule (see (2.25) in [6]) is less accurate than that in (2.7), since the corresponding error bound in [6], similar to that in (2.8), contains the expression $e^{-\pi d / h} /\left(1-e^{-\pi d / h}\right)$ instead of $e^{-2 \pi d / h} /\left(1-e^{-2 \pi d / h}\right)$.

It is important to observe that $N(F, D, \lambda)$ appearing in the inequality (2.8), and defined by (2.9), becomes, in general, unbounded as $\lambda \rightarrow c$, where $c=$ $a$ or $b$. This will occur, for instance, when $c$ is a finite complex number and $F(c) \neq 0$ or $|F(z)| \rightarrow \infty$ as $z \rightarrow c$. However, in this case, typically $|\mathscr{H}(F ; \lambda)| \rightarrow \infty$ as $\lambda \rightarrow c$. If, in addition, $|\mathscr{H}(F ; \lambda)|$ grows at the same rate as $N(F, D, \lambda)$, then it follows from (2.8) that for $\lambda$ near $c$ the relative error $\left|\left[\mathscr{H}(F ; \lambda)-\mathscr{H}_{-\infty}^{\infty}(F ; \lambda)\right] / \mathscr{H}(F ; \lambda)\right|$ will remain small for sufficiently small $h$.

Even though the quadrature rule (2.7) involves infinite series, it is interesting to note its similarity to Hunter's rule (see $[10,4])$. Observe that $\mathscr{H}_{-\infty}^{\infty}(F ; \lambda)$ for $\lambda \neq z_{l}, l \in \mathbb{Z}$, can be regarded as the Sinc quadrature rule (see (4.31) in [13]) applied to $F(z) /(z-\lambda)$, plus one extra term $T(\lambda)=\pi \cot [\pi \phi(\lambda) / h] F(\lambda)$. Note also that if $\lambda=\psi[(m+1 / 2) h]$ for some $m \in \mathbb{Z}$, then $T(\lambda)=0$. This kind of property of quadrature rules for approximating $\mathscr{H}(F ; \lambda)$ has proved important in applications to the numerical solution of singular integral equations by means of quadrature methods (see [9] for references).

One major drawback of the quadrature rule (2.7) is that it requires summation of an infinite series. However, as the next theorem shows, a finite number of 
terms in the series may be sufficient to obtain an approximation to $\mathscr{H}(F ; \lambda)$ within a required error tolerance.

Theorem 2.2. Let all assumptions of Theorem 2.1 be satisfied, and let $\Gamma \subset \mathbb{R}$. Let

$$
|F(z)| \leq C_{j}\left|\phi^{\prime}(z)\right| e^{-\alpha_{j}|\phi(z)|}, \quad z \in \Gamma_{j}, j=1,2,
$$

where $C_{j}$ and $\alpha_{j}, j=1,2$, are positive constants, and where

$$
\Gamma_{1}=\{\psi(x): x \in(-\infty, 0)\}, \quad \Gamma_{2}=\{\psi(x): x \in(0, \infty)\} .
$$

Assume $\lambda \in\left(z_{l-1}, z_{l}\right]$ for some nonpositive $l \in \mathbb{Z}$ or $\lambda \in\left[z_{l}, z_{l+1}\right)$ for some nonnegative $l \in \mathbb{Z}$. Let $N_{1}$ and $N_{2}$ be positive integers such that $-N_{1} \leq l$ if $l \leq 0$ and $N_{2} \geq l$ if $l \geq 0$, and let

$$
\mathscr{H}_{-N_{1}}^{N_{2}}(F ; \lambda)=\left\{\begin{array}{cc}
h \sum_{n=-N_{1}}^{N_{2}} \frac{F\left(z_{n}\right)}{\phi^{\prime}\left(z_{n}\right)\left(z_{n}-\lambda\right)}+\pi \cot \left[\frac{\pi}{h} \phi(\lambda)\right] F(\lambda) & \text { if } \lambda \neq z_{l}, \\
h \sum_{n=-N_{1}}^{N_{2}} \frac{F\left(z_{n}\right)}{\phi^{\prime}\left(z_{n}\right)\left(z_{n}-\lambda\right)}-\frac{h \phi^{\prime \prime}(\lambda)}{2\left[\phi^{\prime}(\lambda)\right]^{2}} F(\lambda) & +\frac{h}{\phi^{\prime}(\lambda)} F^{\prime}(\lambda) \quad \text { if } \lambda=z_{l} .
\end{array}\right.
$$

Then

$$
\begin{aligned}
\left|\mathscr{H}(F ; \lambda)-\mathscr{H}_{-N_{1}}^{N_{2}}(F ; \lambda)\right| \leq & N(F, D, \lambda) \frac{e^{-2 \pi d / h}}{1-e^{-2 \pi d / h}} \\
& +\frac{C_{1} e^{-\alpha_{1} N_{1} h}}{\alpha_{1}\left(\lambda-z_{-N_{1}-1}\right)}+\frac{C_{2} e^{-\alpha_{2} N_{2} h}}{\alpha_{2}\left(z_{N_{2}+1}-\lambda\right)} .
\end{aligned}
$$

Proof. It is easy to see that (2.7), (2.13), and (2.11) yield

$$
\begin{aligned}
\left|\mathscr{H}_{-\infty}^{\infty}(F ; \lambda)-\mathscr{H}_{-N_{1}}^{N_{2}}(F ; \lambda)\right| \leq & \frac{C_{1} h}{\lambda-z_{-N_{1}-1}} \sum_{n \geq N_{1}+1} e^{-\alpha_{1} n h} \\
& +\frac{C_{2} h}{z_{N_{2}+1}-\lambda} \sum_{n \geq N_{2}+1} e^{-\alpha_{2} n h} .
\end{aligned}
$$

Thus, (2.14) follows from the triangle inequality, (2.8), and (2.15), on summing the series in (2.15) and on using the inequalities $e^{\alpha_{j} h}-1 \geq \alpha_{j} h, j=1,2$.

Although $\Gamma$ in Theorem 2.2 is assumed to be a subset of $\mathbb{R}$, a similar result is easily obtained for a more general arc $\Gamma$ in the complex plane. 
In practice, for a given $\lambda \in \Gamma$, the parameters $h, N_{1}$, and $N_{2}$ should be selected so that all three terms in the sum on the right-hand side of (2.14) are sufficiently small and approximately equal to one another. More detailed analysis of how to pick $h, N_{1}, N_{2}$ in the case of $\Gamma=(-1,1)$ is given in $\S 3$.

\section{QUADRATURE RULES OVER $(-1,1)$}

The results of the previous section are used to obtain quadrature rules for the approximate evaluation of Cauchy principal value integrals over the interval $(-1,1)$.

Throughout this section, the arc $\Gamma$ is the interval $(-1,1)$ and the domain $D$ is defined by

$$
D=\{z \in \mathbb{C}:|\arg ((1+z) /(1-z))|<d\}, \quad 0<d<\pi .
$$

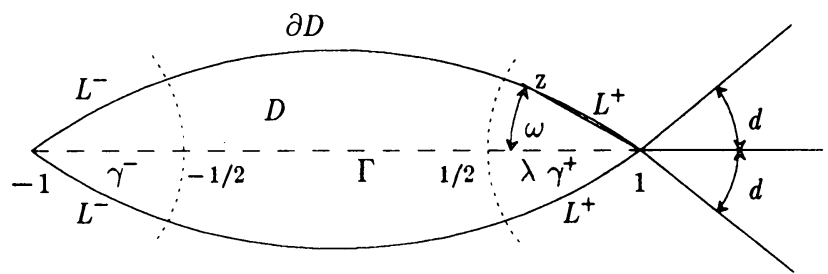

FIGURE 2. The domain $D$

Taking

$$
\phi(z)=\log \left(\frac{1+z}{1-z}\right), \quad \psi(z)=\frac{e^{z}-1}{e^{z}+1},
$$

it is easy to see that Assumption 2.1 is satisfied. In what follows, for $h>0$, the points $z_{n}$ and $z_{n+1 / 2}$ are given by

$$
z_{n}=\frac{e^{n h}-1}{e^{n h}+1}, \quad z_{n+1 / 2}=\frac{e^{(n+1 / 2) h}-1}{e^{(n+1 / 2) h}+1}, \quad n \in \mathbb{Z} .
$$

Note that $z_{n}$ and $z_{n+1 / 2}$ cluster at $\mp 1$ as $n \rightarrow \mp \infty$. For example, if $h=1 / 2$, then $z_{4}=0.7615 \ldots, z_{8}=0.9640 \ldots, z_{16}=0.9993 \ldots$.

The following result is readily obtained from Theorem 2.2.

Theorem 3.1. Let $F \in B(D)$, and let

$$
|F(z)| \leq \begin{cases}C_{1}(1+z)^{\alpha_{1}-1}, & z \in(-1,0), \\ C_{2}(1-z)^{\alpha_{2}-1}, & z \in(0,1),\end{cases}
$$

where $C_{j}$ and $\alpha_{j}, j=1,2$, are positive constants. Assume $\lambda \in\left(z_{l-1}, z_{l}\right]$ for some nonpositive $l \in \mathbb{Z}$ or $\lambda \in\left[z_{l}, z_{l+1}\right)$ for some nonnegative $l \in \mathbb{Z}$. Let $N_{1}$ and $N_{2}$ be positive integers such that $-N_{1} \leq l$ if $l \leq 0$ and $N_{2} \geq l$ if $l \geq 0$, 
and let

$$
\mathscr{H}_{-N_{1}}^{N_{2}}(F ; \lambda)=\left\{\begin{array}{c}
\frac{h}{2} \sum_{n=-N_{1}}^{N_{2}} \frac{1-z_{n}^{2}}{z_{n}-\lambda} F\left(z_{n}\right)+\pi \cot \left[\frac{\pi}{h} \log \left(\frac{1+\lambda}{1-\lambda}\right)\right] F(\lambda) \\
\text { if } \lambda \neq z_{l}, \\
\frac{h}{2} \sum_{\substack{n=-N_{1} \\
n \neq l}}^{N_{2}} \frac{1-z_{n}^{2}}{z_{n}-\lambda} F\left(z_{n}\right)-\frac{h}{2} \lambda F(\lambda) \\
+\frac{h}{2}\left(1-\lambda^{2}\right) F^{\prime}(\lambda) \text { if } \lambda=z_{l} .
\end{array}\right.
$$

Then

$$
\begin{aligned}
\left|\mathscr{H}(F ; \lambda)-\mathscr{H}_{-N_{1}}^{N_{2}}(F ; \lambda)\right| \leq & N(F, D, \lambda) \frac{e^{-2 \pi d / h}}{1-e^{-2 \pi d / h}} \\
& +\frac{C_{1} 2^{\alpha_{1}} e^{-\alpha_{1} N_{1} h}}{\alpha_{1}\left(\lambda-z_{-N_{1}-1}\right)}+\frac{C_{2} 2^{\alpha_{2}} e^{-\alpha_{2} N_{2} h}}{\alpha_{2}\left(z_{N_{2}+1}-\lambda\right)} .
\end{aligned}
$$

Proof. Note that (3.4) and (3.2) imply (2.11) with $C_{1}$ and $C_{2}$ in (2.11) being replaced with $C_{1} 2^{\alpha_{1}}$ and $C_{2} 2^{\alpha_{2}}$, respectively. Thus (3.6) follows directly from (2.14).

Let us briefly outline how $(3.5)$ can be used in practice to compute $\mathscr{H}(F ; \lambda)$ when $|\lambda|$ is not "too close" to 1 . It is clear from (3.6) that for fixed $h$, the positive integers $N_{1}$ and $N_{2}$ should be selected so that all three terms in the sum on the right-hand side of (3.6) are approximately of the same order of magnitude. This leads to $e^{-2 \pi d / h}=e^{-\alpha_{1} N_{1} h}=e^{-\alpha_{2} N_{2} h}$. Hence,

$$
N_{1}=\left\lceil 2 \pi d /\left(\alpha_{1} h^{2}\right)\right\rceil, \quad N_{2}=\left\lceil 2 \pi d /\left(\alpha_{2} h^{2}\right)\right\rceil,
$$

where $\lceil x\rceil$, for real $x$, denotes the smallest integer $\geq x$. It is important to note that if $\lambda$ is close to a point $z_{l}$, then $\mathscr{H}_{-N_{1}}^{N_{2}}(F ; \lambda)$ is likely to produce erroneous results in floating-point arithmetic, owing to loss of significance. Therefore, it is recommended that $\mathscr{H}_{-N_{1}}^{N_{2}}(F ; \lambda)$ be only used when $\lambda$ is sufficiently far away from the points $z_{n}$.

The next theorem specifies conditions under which the bound on $\mid \mathscr{H}(F ; \lambda)-$ $\mathscr{H}_{-N_{1}}^{N_{2}}(F ; \lambda) \mid$ is independent of $\lambda$. This is important for the purpose of solving Cauchy singular integral equations by means of a quadrature method based on the rule (3.5).

Theorem 3.2. Assume $F$ is an analytic function in $D$ such that

$$
|F(z)| \leq C|1+z|^{\alpha_{1}}|1-z|^{\alpha_{2}}, \quad z \in D,
$$

where $C, \alpha_{1}$, and $\alpha_{2}$ are positive constants. Let $\alpha=\min \left(\alpha_{1}, \alpha_{2}\right)$, let $N$ be a positive integer, let $h, N_{1}, N_{2}$ be selected by

$$
h=[2 \pi d /(\alpha N)]^{1 / 2}, \quad N_{j}=\left\lceil\alpha N / \alpha_{j}\right\rceil, \quad j=1,2,
$$


and let $\Gamma^{*}=\left[z_{-N_{1}-1 / 2}, z_{N_{2}+1 / 2}\right] \cup\{-1,1\}$. Let the quadrature rule $\mathscr{H}_{-N_{1}}^{N_{2}}(F ; \lambda)$ for the approximate evaluation of $\mathscr{H}(F ; \lambda), \lambda \in \Gamma^{*}$, be defined by

$$
\mathscr{U}_{-N_{1}}^{N_{2}}(F ; \lambda)=\left\{\begin{aligned}
& \frac{h}{2} \sum_{n=-N_{1}}^{N_{2}} \frac{1-z_{n}^{2}}{z_{n}-\lambda} F\left(z_{n}\right)+ \pi \cot \left[\frac{\pi}{h} \log \left(\frac{1+\lambda}{1-\lambda}\right)\right] F(\lambda) \\
& \text { if } \lambda \in\left[z_{-N_{1}-1 / 2}, z_{N_{2}+1 / 2}\right] \text { and } \\
& \lambda \neq z_{l} \text { for all } l,-N_{1} \leq l \leq N_{2}, \\
& \frac{h}{2} \sum_{\substack{N_{2} \\
n \neq l}}^{N_{1}} \frac{1-z_{n}^{2}}{z_{n}-\lambda} F\left(z_{n}\right)-\frac{h}{2} \lambda F(\lambda)+\frac{h}{2}\left(1-\lambda^{2}\right) F^{\prime}(\lambda) \\
& \text { if } \lambda \in\left[z_{-N_{1}-1 / 2}, z_{N_{2}+1 / 2}\right] \text { and } \\
& \lambda=z_{l} \text { for some } l,-N_{1} \leq l \leq N_{2}, \\
& \frac{h}{2} \sum_{n=-N_{1}}^{N_{2}} \frac{1-z_{n}^{2}}{z_{n}-\lambda} F\left(z_{n}\right) \text { if } \lambda=\mp 1 .
\end{aligned}\right.
$$

Then for all $\lambda \in \Gamma^{*}$,

$$
\begin{aligned}
& \left|\mathscr{H}(F ; \lambda)-\mathscr{H}_{-N_{1}}^{N_{2}}(F ; \lambda)\right| \\
& \quad \leq C^{\prime} \begin{cases}N^{1 / 2} e^{-(2 \pi d \alpha N)^{1 / 2}} & \text { if } \lambda \in\left[z_{-N_{1}-1 / 2}, z_{N_{2}+1 / 2}\right], \\
e^{-(2 \pi d \alpha N)^{1 / 2}} & \text { if } \lambda=\mp 1,\end{cases}
\end{aligned}
$$

where $C^{\prime}$ is a positive constant which depends only on $D, C, \alpha_{1}$, and $\alpha_{2}$.

The proof of the theorem is based on the following two lemmas.

Lemma 3.1. Let $\alpha_{1}$ and $\alpha_{2}$ be positive constants. Then

$$
M\left(D, \alpha_{1}, \alpha_{2}\right) \equiv \sup _{\lambda \in \Gamma} \int_{\partial D} \frac{|1+z|^{\alpha_{1}}|1-z|^{\alpha_{2}}}{|z-\lambda|}|d z|<\infty .
$$

Proof. Lemma 5.4 of [1] could be used to establish (3.12). Here, a more direct proof will be presented. Clearly, setting $\alpha=\min \left(\alpha_{1}, \alpha_{2}\right)$, it will be enough to verify that

$$
\sup _{\lambda \in \Gamma} \int_{\partial D} \frac{\left|1-z^{2}\right|^{\alpha}}{|z-\lambda|}|d z|<\infty .
$$

Let $\Gamma^{\prime}=\gamma^{-} \cup \gamma^{+}$, where $\gamma^{ \pm}=\{\lambda \in \Gamma:|\lambda \mp 1| \leq 1 / 2\}$, and similarly let $\partial D^{\prime}=L^{-} \cup L^{+}$, where $L^{ \pm}=\{z \in \partial D:|z \mp 1| \leq 1 / 2\}$ (see Figure 2). It will be sufficient to show that $(3.13)$ is satisfied with $\Gamma$ and $\partial D$ replaced by $\Gamma^{\prime}$ and $\partial D^{\prime}$, respectively. For definiteness replace $\Gamma$ with $\gamma^{+}$and $\partial D$ with $L^{+}$ 
(all other cases can be treated analogously). Let $\lambda \in \gamma^{+}$, let $z \in L^{+}$, and let $\omega$ be the angle between the line segments $1 \lambda$ and $1 z$. Inspecting the triangle $\lambda 1 z$ (see Figure 2), it is easy to see that there exists an angle $\omega^{*}, 0<\omega^{*}<\omega$, such that

$$
|z-1| \leq \frac{|z-\lambda|}{\sin (\omega)} \leq \frac{|z-\lambda|}{\sin \left(\omega^{*}\right)}
$$

for all $\lambda \in \gamma^{+}$and all $z \in L^{+}$. Thus it follows from (3.14) that

$$
\sup _{\lambda \in \gamma^{+}} \int_{L^{+}} \frac{\left|1-z^{2}\right|^{\alpha}}{|z-\lambda|}|d z| \leq \frac{1}{\sin \left(\omega^{*}\right)} \int_{L^{+}}\left|1-z^{2}\right|^{\alpha-1}|d z|<\infty,
$$

which completes the proof of the lemma.

Lemma 3.2. Let $\lambda \in \Gamma \backslash\left(z_{n-1 / 2}, z_{n+1 / 2}\right)$. Then

$$
\frac{h}{2} \frac{1-z_{n}^{2}}{\left|z_{n}-\lambda\right|} \leq 2 e^{h / 2} \text {. }
$$

Proof. (3.16) follows easily from (3.3) (cf. also the proof of Lemma 2.11 in [6]).

Proof of Theorem 3.2. Clearly $F \in B(D)$, and hence all assumptions of Theorem 2.1 are satisfied. First, consider the case when $\lambda \in\left[z_{-N_{1}-1 / 2}, z_{N_{2}+1 / 2}\right]$. Using (3.16) and (3.8), it is easy to see that

$$
\begin{aligned}
& \frac{h}{2}\left(\sum_{n \leq-N_{1}-1}+\sum_{n \geq N_{2}+1}\right) \frac{1-z_{n}^{2}}{\left|z_{n}-\lambda\right|}\left|F\left(z_{n}\right)\right| \\
& \quad \leq \frac{C 2^{\alpha_{1}+\alpha_{2}+1} e^{h / 2}}{h}\left(\frac{e^{-\alpha_{1} N_{1} h}}{\alpha_{1}}+\frac{e^{-\alpha_{2} N_{2} h}}{\alpha_{2}}\right) .
\end{aligned}
$$

Further, the triangle inequality, (2.8), (2.9), (3.8), (3.12), and (3.17) yield

$$
\begin{aligned}
\left|\mathscr{H}(F ; \lambda)-\mathscr{H}_{-N_{1}}^{N_{2}}(F ; \lambda)\right| \leq & C M\left(D, \alpha_{1}, \alpha_{2}\right) \frac{e^{-2 \pi d / h}}{1-e^{-2 \pi d / h}} \\
& +\frac{C 2^{\alpha_{1}+\alpha_{2}+1} e^{h / 2}}{h}\left(\frac{e^{-\alpha_{1} N_{1} h}}{\alpha_{1}}+\frac{e^{-\alpha_{2} N_{2} h}}{\alpha_{2}}\right) .
\end{aligned}
$$

The inequality (3.11) for $\lambda \in\left[z_{-N_{1}-1 / 2}, z_{N_{2}+1 / 2}\right]$ follows now from (3.18) and (3.9). If $\lambda=1$ (the case of $\lambda=-1$ is analogous), then in place of (3.18) one gets

$$
\begin{aligned}
\left|\mathscr{H}(F ; \lambda)-\mathscr{H}_{-N_{1}}^{N_{2}}(F ; \lambda)\right| \leq & C M\left(D, \alpha_{1}, \alpha_{2}\right) \frac{e^{-2 \pi d / h}}{1-e^{-2 \pi d / h}} \\
& +C 2^{\alpha_{1}+\alpha_{2}}\left(\frac{e^{-\left(\alpha_{1}+1\right) N_{1} h}}{\alpha_{1}+1}+\frac{e^{-\alpha_{2} N_{2} h}}{\alpha_{2}}\right),
\end{aligned}
$$

and (3.11) is obtained from (3.9). 
The quadrature rule (3.10) can again be compared with the corresponding rule in $(2.50)$ of [6]. Since the error bound (3.11) contains the term $e^{-(2 \pi d \alpha N)^{1 / 2}}$, whereas the similar error bound in [6] involves $e^{-(\pi d \alpha N)^{1 / 2}}$ instead, the quadrature rule (3.10) is expected to be more accurate for $\lambda \in \Gamma^{*}$. It is also interesting to note that if $\lambda=z_{m+1 / 2},-N_{1}-1 \leq m \leq N_{2}$, then the quadrature rule (3.10) coincides with that in (2.50) of [6], since in this case the term involving $F(\lambda)$ drops out of the first formula in (3.10).

\section{AN APPLICATION TO THE APPROXIMATE SOLUTION OF CAUCHY SINGULAR INTEGRAL EQUATIONS}

In this section the Sinc quadrature rule (3.10) is used to obtain an approximate solution to a Cauchy singular integral equation (CSIE). Quadrature methods for CSIE's have been extensively studied by many authors (see [9] for references). In the majority of these methods, nodes as well as collocation points are zeros of corresponding orthogonal polynomials. Recently, in [14], an attempt has been made to directly approximate CSIE by means of one of the Sinc quadrature rules derived by Stenger in [12]. It is believed by this author that the approach used here is more natural and straightforward than that of [14]. Moreover, the convergence analysis presented here, although still incomplete from the theoretical point of view, shows better why the Sinc quadrature method based on (3.10) works.

Consider the CSIE of the form

$$
f_{-1}^{1} \frac{\left(1-\tau^{2}\right)^{-1 / 2} w(\tau)}{\tau-t} d \tau=f(t), \quad t \in(-1,1),
$$

whose solution $w$ is subject to the condition

$$
\int_{-1}^{1}\left(1-\tau^{2}\right)^{-1 / 2} w(\tau) d \tau=c
$$

In (4.1) and (4.2), $f$ and $c$ are a given function and constant, respectively. To describe a Sinc quadrature method of solving (4.1) and (4.2), assume that $w$ satisfies the following conditions.

Assumption 4.1. Let $w$ be an analytic function in $D$ (see (3.1)), and let there exist positive constants $C, \alpha_{1}$, and $\alpha_{2}$ such that

$$
\begin{aligned}
& \mid w(z)-\frac{1-z}{2} w(-1)-\frac{1+z}{2} w(1) \mid \\
& \leq C|1+z|^{1 / 2+\alpha_{1}}|1-z|^{1 / 2+\alpha_{2}}, \quad z \in D .
\end{aligned}
$$

Rewriting the solution $w$ in the form

$$
w(\tau)=\left(1-\tau^{2}\right)^{1 / 2} g(\tau)+\frac{1-\tau}{2} w(-1)+\frac{1+\tau}{2} w(1), \quad \tau \in[-1,1],
$$

and substituting it back into (4.1) and (4.2) yields

$$
-\frac{\pi}{2} w(-1)+f_{-1}^{1} \frac{g(\tau)}{\tau-t} d \tau+\frac{\pi}{2} w(1)=f(t), \quad t \in(-1,1),
$$


and

$$
\frac{\pi}{2} w(-1)+\int_{-1}^{1} g(\tau) d \tau+\frac{\pi}{2} w(1)=c .
$$

Thus, the original problem has been reduced to finding $w(-1), w(1)$, and $g$ which satisfy (4.5) and (4.6). Once $w(-1), w(1)$, and $g$ are known, the solution $w$ of (4.1) and (4.2) is obtained by (4.4).

It follows from Assumption 4.1 that $g$ of (4.4) satisfies all hypotheses of Theorem 3.2. Let $N$ be a positive integer, and let $h, N_{1}$, and $N_{2}$ be selected as in (3.9), where $\alpha=\min \left(\alpha_{1}, \alpha_{2}\right)$. Let the points $z_{n}, n=-N_{1}(1) N_{2}$, and $z_{n+1 / 2}, n=-N_{1}(1) N_{2}-1$, be given by (3.3). To simplify upcoming notation, assume also that $z_{-N_{1}-1 / 2}=z_{-N_{1}-1}=-1$, and that $z_{N_{2}+1 / 2}=z_{N_{2}+1}=1$. The Sinc quadrature method of this section consists in carrying out the following steps. First, replace the Cauchy principal value integral in (4.5) with the quadrature rule (3.10), and then collocate (4.5) at $t=z_{m+1 / 2}, m=-N_{1}-1(1) N_{2}$. Similarly, apply the quadrature rule of (4.32) in [13] to the integral in (4.6). This procedure yields the equations

$$
\begin{array}{r}
-\frac{\pi}{2} w(-1)+\frac{h}{2} \sum_{n=-N_{1}}^{N_{2}} \frac{1-z_{n}^{2}}{z_{n}-z_{m+1 / 2}} g\left(z_{n}\right)+\frac{\pi}{2} w(1) \\
=f\left(z_{m+1 / 2}\right)+\varepsilon_{m}, \quad m=-N_{1}-1(1)-1,
\end{array}
$$

$$
\frac{\pi}{2} w(-1)+\frac{h}{2} \sum_{n=-N_{1}}^{N_{2}}\left(1-z_{n}^{2}\right) g\left(z_{n}\right)+\frac{\pi}{2} w(1)=c+\varepsilon_{0}
$$

$$
\begin{gathered}
-\frac{\pi}{2} w(-1)+\frac{h}{2} \sum_{n=-N_{1}}^{N_{2}} \frac{1-z_{n}^{2}}{z_{n}-z_{m-1 / 2}} g\left(z_{n}\right)+\frac{\pi}{2} w(1) \\
=f\left(z_{m-1 / 2}\right)+\varepsilon_{m}, \quad m=1(1) N_{2}+1
\end{gathered}
$$

It follows from (3.11), and from (4.32) in [13], that the error terms $\varepsilon_{m}$ in (4.7)-(4.9) satisfy

$$
\left|\varepsilon_{m}\right|=O\left(N^{1 / 2} e^{-(2 \pi d \alpha N)^{1 / 2}}\right), \quad m=-N_{1}-1(1) N_{2}+1
$$

Let $A_{N}=\left(a_{m, n}\right)_{m, n=-N_{1}-1(1) N_{2}+1}$ be the square coefficient matrix of order 
$N_{1}+N_{2}+3$ whose elements $a_{m, n}$ are given by

(4.11) $a_{m, n}= \begin{cases}-\frac{\pi}{2} & \text { if } m=-N_{1}-1(1)-1,1(1) N_{2}+1 \\ \frac{\pi}{2} & \text { and } n=-N_{1}-1, \\ \frac{h}{2} \frac{1-z_{n}^{2}}{z_{n}-z_{m+1 / 2}} & \text { if } m=0 \text { and } n=-N_{1}-1 \\ \frac{h}{2}\left(1-z_{n}^{2}\right) & \text { if } m=0 \text { and } n=-N_{1}(1) N_{2}, \\ \frac{h}{2} \frac{1-z_{n}^{2}}{z_{n}-z_{m-1 / 2}} & \text { if } m=1(1) N_{2}+1 \text { and } n=N_{2}+1,\end{cases}$

Furthermore, let $\mathbf{f}_{N}$ be the vector defined by

$\mathbf{f}_{N}=\left(f(-1), f\left(z_{-N_{1}+1 / 2}\right), \ldots, f\left(z_{-1 / 2}\right), c, f\left(z_{1 / 2}\right), \ldots, f\left(z_{N_{2}-1 / 2}\right), f(1)\right)^{T}$.

Dropping the error terms $\varepsilon_{m}$ in (4.7)-(4.9) gives rise to the system of linear equations

$$
A_{N} \mathbf{g}_{N}=\mathbf{f}_{N},
$$

where the vector $\mathbf{g}_{N}$ is of the form

$$
\mathbf{g}_{N}=\left(g_{-N_{1}-1}, g_{-N_{1}}, \ldots, g_{N_{2}}, g_{N_{2}+1}\right)^{T} \text {. }
$$

It is hoped, of course, that the components $g_{n}, n=-N_{1}-1(1) N_{2}+1$, of $\mathbf{g}_{N}$ will approximate $w(-1), g\left(z_{n}\right), n=-N_{1}(1) N_{2}$, and $w(1)$, respectively.

Using (3.16), it is easy to see that

$$
\left\|A_{N}\right\|_{\infty} \leq \pi+2\left(N_{1}+N_{2}+1\right) e^{h / 2} .
$$

At present, proving nonsingularity of $A_{N}$ and finding an upper bound on $\left\|A_{N}^{-1}\right\|_{\infty}$ appear to be challenging problems. Nevertheless, numerical tests indicate that $A_{N}^{-1}$ exists and, moreover, $\left\|A_{N}^{-1}\right\|_{\infty}$ seems to depend very weakly on the order of $A_{N}$. For several values of $d / \alpha$ and $N$, with $\alpha_{1}=\alpha_{2}=\alpha$, the results of the tests are given in Table 1.

\begin{tabular}{|c|c|c|c|c|c|c|c|c|c|}
\hline$d / \alpha$ & \multicolumn{3}{|c|}{$\pi / 2$} & \multicolumn{3}{|c|}{$\pi$} & \multicolumn{3}{|c|}{$2 \pi$} \\
\hline$N$ & 8 & 16 & 32 & 8 & 16 & 32 & 8 & 16 & 32 \\
\hline$\left\|A_{N}\right\|_{\infty}$ & 14.8 & 19.2 & 24.9 & 17.8 & 23.7 & 31.7 & 22.1 & 30.3 & 41.5 \\
\hline$\left\|A_{N}^{-1}\right\|_{\infty}$ & 1 & 1.2 & 1.5 & .8 & 1 & 1.2 & .7 & .8 & 1 \\
\hline
\end{tabular}

TABLE 1

Values of $\left\|A_{N}\right\|_{\infty}$ and $\left\|A_{N}^{-1}\right\|_{\infty}$ 
Once (4.13) has been solved, corresponding approximations to $w$ at the points $z_{n}, n=-N_{1}-1(1) N_{2}+1$, are obtained by

$$
\begin{aligned}
w_{n}= & \left(1-z_{n}^{2}\right)^{1 / 2} g_{n}+\frac{1-z_{n}}{2} g_{-N_{1}-1} \\
& +\frac{1+z_{n}}{2} g_{N_{2}+1}, \quad n=-N_{1}-1(1) N_{2}+1 .
\end{aligned}
$$

To analyze the accuracy of these approximations, introduce the vector

$$
\mathbf{e}_{N}=\left(w(-1)-w_{-N_{1}-1}, w\left(z_{-N_{1}}\right)-w_{-N_{1}}, \ldots, w\left(z_{N_{2}}\right)-w_{N_{2}}, w(1)-w_{N_{2}+1}\right)^{T} .
$$

It follows easily from $(4.17),(4.4),(4.16),(4.7)-(4.9),(4.13)$, and (4.10) that

$$
\left\|\mathbf{e}_{N}\right\|_{\infty} \leq\left\|A_{N}^{-1}\right\|_{\infty} O\left(N^{1 / 2} e^{-(2 \pi d \alpha N)^{1 / 2}}\right)
$$

The estimate (4.18) is also obtained if instead of -1 and 1 , the points $z_{-N_{1}-1 / 2}$ and $z_{N_{2}+1 / 2}$, given by (3.3), are used as collocation points. The results reported in Table 1 and (4.18) indicate a very rapid rate of convergence of the Sinc quadrature method. Recall that if $w^{\prime}$ satisfies a Lipschitz condition on $[-1,1]$ with exponent $\mu$, then the discrete error of the $n$-point Gauss quadrature method for solving (4.1), (4.2) is of order $O\left(n^{-\mu} \log n\right)$ as $n \rightarrow \infty$ (see [5]). In comparison, the Sinc quadrature method yields (4.18) for $w$ satisfying Assumption 4.1. Note that this assumption allows $w^{\prime}$ to be unbounded at the endpoints of $[-1,1]$.

The Sinc quadrature method presented here can be easily extended to a general CSIE of the first kind,

$$
\begin{array}{r}
f_{-1}^{1} \frac{\left(1-\tau^{2}\right)^{-1 / 2} w(\tau)}{\tau-t} d \tau+\int_{-1}^{1} K(t, \tau)\left(1-\tau^{2}\right)^{-1 / 2} w(\tau) d \tau=f(t) \\
t \in(-1,1)
\end{array}
$$

where as before $w$ is subject to (4.2). In this case, the integral involving $K$ is approximated, similarly as (4.2) before, by the quadrature rule (4.32) of [13].

It was demonstrated in $\S 3$ that the points $z_{n}$ and $z_{m+1 / 2}$ cluster at \pm 1 as $n, m \rightarrow \pm \infty$. Therefore, computing first $z_{n}$ and $z_{m+1 / 2}$ by (3.3), and then using these values to obtain the coefficients (4.11) of the matrix $A_{N}$ may lead to loss of significance in floating-point arithmetic. This difficulty is easily remedied by substituting (3.3) into the formulas defining $a_{m, n}$ and simplifying the resulting expressions. For instance, it is easy to see that

$$
\frac{1-z_{n}^{2}}{z_{n}-z_{m \pm 1 / 2}}=\frac{2\left(e^{(m \pm 1 / 2) h}+1\right)}{\left(e^{n h}+1\right)\left(1-e^{(m-n \pm 1 / 2) h}\right)} .
$$

The expression on the right-hand side in (4.20) provides a numerically stable way of carrying out computations. Note, e.g., that if $d=\pi / 2, \alpha_{1}=\alpha_{2}=1 / 2$, and $N=32$, then, by (3.9), $h=\pi / 4$. Thus, there will be practically no loss of significant digits in subtracting $e^{(m-n \pm 1 / 2) h}$ from 1 . 


\section{NuMERICAL EXAMPLES}

Examples presented in this section illustrate the superiority of the Sinc quadrature rule over Gauss type and piecewise-polynomial quadrature rules in the case of badly behaved integrands.

Example 1. For several values of $\lambda \in(-1,1)$, the Sinc quadrature rule (3.5) was used to evaluate

$$
\mathscr{H}(F ; \lambda)=f_{-1}^{1} \frac{(1+z)^{-1 / 4}(1-z)^{1 / 4}}{z-\lambda} d z=\pi(1+\lambda)^{-1 / 4}(1-\lambda)^{1 / 4}-\pi \sqrt{2}
$$

Parameters $d$ and $\alpha_{1}, \alpha_{2}$ (see (3.1) and (3.4)) were selected as $\pi / 2$ and $3 / 4$, $5 / 4$, respectively. For a given $h$, integers $N_{1}, N_{2}$ were determined by (3.7). Numerical results of the tests are presented in Table 2 (integers in parentheses denote decimal exponents).

TABLE 2

The error $\varepsilon(\lambda)=\left|\mathscr{H}(F ; \lambda)-\mathscr{H}_{-N_{1}}^{N_{2}}(F ; \lambda)\right|$ for $(5.1)$

\begin{tabular}{c|c|c|c|c|c|c|c|c|c}
\hline$h$ & $N_{1}$ & $N_{2}$ & $e^{-2 \pi d / h}$ & $\varepsilon(-.8)$ & $\varepsilon(-.3)$ & $\varepsilon(0)$ & $\varepsilon(.1)$ & $\varepsilon(.6)$ & $\varepsilon(.9)$ \\
\hline 1 & 14 & 8 & $.5(-4)$ & $.2(-3)$ & $.4(-4)$ & $.1(-4)$ & $.4(-5)$ & $.6(-4)$ & $.3(-3)$ \\
$1 / 2$ & 53 & 32 & $.3(-8)$ & $.2(-7)$ & $.6(-8)$ & $.3(-8)$ & $.2(-8)$ & $.3(-8)$ & $.2(-7)$ \\
$1 / 3$ & 119 & 72 & $.1(-12)$ & $.1(-11)$ & $.3(-12)$ & $.2(-12)$ & $.1(-12)$ & $.1(-12)$ & $.1(-11)$ \\
\hline
\end{tabular}

For all listed values of $\lambda$, except $\lambda=0$, the first formula of (3.5) was used to evaluate $\mathscr{H}(F ; \lambda)$. Since $z_{0}=0$ (see (3.3)), the second formula in (3.5) was employed to compute $\mathscr{H}(F ; 0)$. Analyzing the entries of Table 2 , it is seen that the errors remain within the error bound given by (3.6). Recently, a very accurate, although somewhat involved, method was proposed in [7] to evaluate $\mathscr{H}(F ; \lambda)$ for $F(z)=(1+z)^{\beta_{1}}(1-z)^{\beta_{2}}, \beta_{1}, \beta_{2}>-1$. Obviously, the Sinc quadrature rule (3.5) can be applied to more general functions $F$ such as

$$
F(z)=(1+z)^{-1 / 3}(1-z)^{-1 / 4} \log (1-z)
$$

or

$$
F(z)=(1+z)^{-1 / 5}(1-z)^{-1 / 2} e^{(1+z)^{1 / 3}(1-z)^{1 / 4}} .
$$

Example 2. The following CSIE was solved in $[15,8]$ :

$$
\sin \frac{\pi t}{2} \Omega(t) w(t)+\frac{1}{\pi} f_{-1}^{1} \frac{\cos (\pi \tau / 2) \Omega(\tau) w(\tau)}{\tau-t} d \tau=f(t), \quad
$$

where

$$
\Omega(t)=e\left(1-t^{2}\right)^{-1 / 2}(1-t)^{t / 2}(1+t)^{-t / 2}, \quad e=2.71828 \ldots
$$


The solution $w$ of (5.2) can be expressed in closed form,

$$
w(t)=\frac{\sin (\pi t / 2) f(t)}{\Omega(t)}-\frac{1}{\pi} f_{-1}^{1} \frac{\cos (\pi \tau / 2) f(\tau)}{\Omega(\tau)(\tau-t)} d \tau+c,
$$

where $c$ is an arbitrary constant. Consider a particular solution $w$ corresponding to $f(t)=\left(1-t^{2}\right)^{1 / 2}$ and $c=1 / \pi$. It follows from (5.4) and (5.3) that the Cauchy principal value integral

$$
f_{-1}^{1} \frac{\left(1-\tau^{2}\right)^{1 / 2} G(\tau)}{\tau-t} d \tau
$$

where

$$
G(t)=\cos (\pi t / 2)(1-t)^{(1-t) / 2}(1+t)^{(1+t) / 2},
$$

needs to be evaluated. It can be shown that the function $G$ of (5.6) is continuously differentiable on $[-1,1]$ and that its second derivative has logarithmic singularities at \pm 1 . For this reason, the integral (5.5) was evaluated in [8] by means of a piecewise-linear quadrature rule, instead of a Gauss type quadrature corresponding to the weight function $\left(1-\tau^{2}\right)^{1 / 2}$. The rule was based on an $n$-point nonuniform mesh with nodes concentrated at the endpoints \pm 1 . For $t=1$, Gerasoulis found approximations $w_{n}(1)$ to $w(1)=.5185916 \ldots$, and then employed extrapolation to improve his results, which are reported in Table 3.

\section{TABLE 3}

The piecewise-linear quadrature of [8] for (5.4)

\begin{tabular}{c|c|c|c|c}
\hline$n$ & 10 & 20 & 40 & 80 \\
\hline$w_{n}(1)$ & .508712 & .515989 & .517931 & .518426 \\
\hline Extrap & - & .518414 & .518579 & .518592 \\
\hline
\end{tabular}

On the other hand, it is easy to see that the function $F(\tau)=\left(1-\tau^{2}\right)^{1 / 2} G(\tau)$, with $G$ given by (5.6), is analytic in the interior of the unit disc centered at the origin. Moreover, $F$ satisfies (3.8) with $\alpha_{1}=\alpha_{2}=3 / 2$. The Sinc quadrature rule of (3.10), with $d=\pi / 2$, and the selection of $N_{1}$ and $N_{2}$ based on (3.19) (cf. also (3.6) and (3.7)) was used to compute (5.5) for $t=1$. Table 4 presents the approximations $w_{-N_{1}}^{N_{2}}(1)$ to $w(1)$ obtained in this manner.

TABLE 4

The Sinc quadrature (3.10) for (5.4)

\begin{tabular}{c|c|c|c|c}
\hline$N_{1}, N_{2}$ & 2,3 & 4,7 & 8,12 & 16,27 \\
\hline$w_{-N_{1}}^{N_{2}}(1)$ & .5182984 & .5185745 & .5185908 & .5185916 \\
\hline
\end{tabular}


Recall that the Sinc quadrature rule (3.10) requires $N_{1}+N_{2}+1$ function evaluations to obtain $w_{-N_{1}}^{N_{2}}(1)$, whereas the piecewise-linear quadrature rule of [8] uses $n$ such evaluations to produce $w_{n}(1)$. In addition, certain integrals independent of the function $G$ must be computed in advance so that the piecewiselinear quadrature can be applied. Comparing entries of Tables 3 and 4, it is clear that the Sinc quadrature rule is more efficient than the piecewise-linear quadrature rule.

Example 3. Consider the following CSIE (cf. (4.1), (4.2)):

$$
\begin{gathered}
f_{-1}^{1} \frac{\left(1-\tau^{2}\right)^{-1 / 2} w(\tau)}{\tau-t} d \tau=2 t+f(t), \quad t \in(-1,1), \\
\int_{-1}^{1}\left(1-\tau^{2}\right)^{-1 / 2} w(\tau) d \tau=c,
\end{gathered}
$$

where for given $0<\alpha_{1}, \alpha_{2} \leq 1 / 2$,

$$
f(t)=f_{-1}^{1} \frac{(1+\tau)^{\alpha_{1}}(1-\tau)^{\alpha_{2}}}{\tau-t} d \tau, \quad c=\int_{-1}^{1}(1+\tau)^{\alpha_{1}}(1-\tau)^{\alpha_{2}} d \tau .
$$

The solution $w$ of $(5.7)-(5.9)$ is given by

$$
w(\tau)=2 \tau^{2}-1+(1+\tau)^{1 / 2+\alpha_{1}}(1-\tau)^{1 / 2+\alpha_{2}} .
$$

Note, that although $w$ satisfies a Lipschitz condition on $[-1,1], w^{\prime}$ becomes unbounded at \pm 1 if $\alpha_{1}, \alpha_{2}<1 / 2$. On the other hand, it is easy to see that $w$ satisfies Assumption 4.1. The problem (5.7)-(5.9) was solved via the Sinc quadrature method of $\S 4$. When setting up the right-hand side of (4.13), the values of $f$ and $c$ were computed with high accuracy. The approximate solution to $w$ and the error vector $\mathbf{e}_{N}$ were obtained by (4.16) and (4.17), respectively. For $d=\pi / 2$ and various values of $\alpha_{1}, \alpha_{2}$, and $N$, the corresponding values of $\left\|\mathbf{e}_{N}\right\|_{\infty}$ are presented in Table 5.

\section{TABLE 5}

Sinc quadrature method of $\S 4$ for (5.7)-(5.9)

\begin{tabular}{c|c|c|c|c|c|c}
\hline$\alpha_{1}, \alpha_{2}$ & \multicolumn{2}{|c|}{$1 / 2,1 / 2$} & \multicolumn{2}{c|}{$1 / 2,1 / 4$} & \multicolumn{2}{c}{$1 / 4,1 / 4$} \\
\hline$N$ & 4 & 16 & 8 & 32 & 8 & 32 \\
$N_{1}, N_{2}$ & 4,4 & 16,16 & 4,8 & 16,32 & 8,8 & 32,32 \\
$\left\|\mathbf{e}_{N}\right\|_{\infty}$ & $.9(-2)$ & $.5(-5)$ & $.1(-1)$ & $.7(-5)$ & $.1(-1)$ & $.8(-5)$ \\
\hline
\end{tabular}

The above results seem to support the conclusions of $\S 4$ regarding $\left\|A_{N}^{-1}\right\|_{\infty}$ and the rate of convergence of the Sinc quadrature method (see Table 1 and (4.18)). 


\section{BIBLIOGRAPHY}

1. B. Bialecki and F. Stenger, Sinc-Nyström method for numerical solution of one-dimensional Cauchy singular integral equation given on a smooth arc in the complex plane, Math. Comp. 51 (1988), 133-165.

2. B. Bialecki, A modified Sinc-quadrature rule for functions with poles near the arc of integration, BIT 29 (1989), 464-476.

3. __ Sinc-Nyström method for numerical solution of dominant system of Cauchy singular integral equations given on a piecewise smooth contour, SIAM J. Numer. Anal. 26 (1989), 1194-1211.

4. D. Elliott and D. F. Paget, Gauss type quadrature rules for Cauchy principal value integrals, Math. Comp. 33 (1979), 301-309.

5. D. Elliott, The classical collocation method for singular integral equations, SIAM J. Numer. Anal. 19 (1982), 816-832.

6. D. Elliott and F. Stenger, Sinc method of solution of singular integral equations, IMACS Symposium on Numerical Solution of Singular Integral Equations, IMACS, 1984, pp. 2735.

7. W. Gautschi and J. Wimp, Computing the Hilbert transform of a Jacobi weight function, BIT 27 (1987), 203-215.

8. A. Gerasoulis, Piecewise-polynomial quadratures for Cauchy singular integrals, SIAM J. Numer. Anal. 23 (1986), 891-902.

9. M. A. Golberg, The numerical solution of Cauchy singular integral equations with constant coefficients, J. Integral Equations (Suppl.) 9 (1985), 127-151.

10. D. B. Hunter, Some Gauss-type formulae for the evaluation of Cauchy principal values of integrals, Numer. Math. 19 (1972), 419-424.

11. N. I. Muskhelishvili, Singular integral equations, Noordhoff, Groningen, 1958.

12. F. Stenger, Approximations via Whittaker's cardinal function, J. Approx. Theory 17 (1976), 222-240.

13. _ Numerical methods based on Whittaker cardinal, or Sinc functions, SIAM Rev. 23 (1981), 165-224.

14. E. Venturino, On solving integral equations via a hyperbolic tangent quadrature rule, Math. Comp. 47 (1986), 159-167.

15. S. Welstead, Orthogonal polynomials applied to the solution of singular integral equations, Ph.D. Thesis, Purdue Univ., West Lafayette, IN, 1982. 0027

Department of Mathematics, University of Kentucky, Lexington, Kentucky 40506 -

E-mail address: bialecki@ms.uky.edu 